\title{
Effect of Initial Orientation on the Laser-Induced Cycloaddition Reaction of Benzene and Ethylene
}

\author{
Shuai Yuan, ${ }^{1}$ Huiling Hong, ${ }^{1}$ Gang Wang, ${ }^{1}$ Wenying Zhang, ${ }^{1}$ Weifeng Wu, \\ Yusheng Dou, ${ }^{2}$ and Glenn V. Lo $^{2}$ \\ ${ }^{1}$ School of Bioinformation, Chongqing University of Posts and Telecommunications, Chongqing 400065, China \\ ${ }^{2}$ Department of Physical Sciences, Nicholls State University, P.O. Box 2022, Thibodaux, LA 70310, USA
}

Correspondence should be addressed to Shuai Yuan; yuanshuai@cqupt.edu.cn and Yusheng Dou; yusheng.dou@nicholls.edu

Received 7 May 2014; Accepted 18 July 2014; Published 28 October 2014

Academic Editor: Fuli Li

Copyright (C) 2014 Shuai Yuan et al. This is an open access article distributed under the Creative Commons Attribution License, which permits unrestricted use, distribution, and reproduction in any medium, provided the original work is properly cited.

The $[2+2]$ photocycloaddition reaction of benzene and ethylene was investigated by semiclassical dynamics simulation and complete active space self-consistent field (CASSCF) ab initio calculations. Following laser excitation of the benzene molecule, two mechanisms were observed depending on the location of the second $\mathrm{C}$ of ethylene in relation to the hexagonal prism space defined by the first $\mathrm{C}$ and the plane of the benzene ring. Synchronous formation of two bonds was observed when the second $\mathrm{C}$ is outside the prism space; an asynchronous mechanism is observed otherwise. Charge transfer was observed only in the asynchronous mechanism; CASSCF calculations suggest that the asynchronous mechanism involves a barrierless path from the Frank-Condon point to a conical intersection, while the synchronous mechanism involves $0.8 \mathrm{eV}$ barrier. These results are consistent with a higher quantum yield observed in the simulations for the asynchronous pathway.

\section{Introduction}

A photocycloaddition reaction is a light-induced reaction that forms a new molecule from two unsaturated molecules via two atoms from each molecule. These reactions are "atomeconomical" and "step-economical"; all the atoms in the reactants appear in the products and complexity can be achieved in a single step $[1,2]$. With unprecedented possibilities to efficiently create various new molecules, photocycloadditions are widely applied in organic synthesis. A photocycloaddition reaction between benzene (or its derivatives) and an alkene can be classified into three types: [2+2] (or ortho), [3+2] (or $m e t a$ ), and $[4+2]$ (or para). Photocycloadditions of benzene and an alkene are usually induced by the photoexcitation of the benzene molecule. If the $S_{1}$ state of benzene is involved, only the meta-addition mode is allowed to occur in a concerted fashion (i.e., synchronous formation of two single bonds) according to molecular orbital symmetry rules $[3,4]$. The formation of ortho- and para-cycloadducts can be interpreted as being due to charge transfer, a nonconcerted mechanism, or the involvement of the $\mathrm{S}_{2}$ excited state.
Robb's group has conducted theoretical studies of the photocycloaddition of benzene and ethylene system using CASSCF calculation and MMVB simulation [5]. They found that such reactions could occur from $S_{1}$ state barrierless through a conical intersection (CI), which is shared by all three photocycloaddition products. This low energy CI follows the formation of one $\mathrm{C}-\mathrm{C}$ bond between benzene and ethylene. Subsequent studies [6] suggest that the chemical selectivity is, in part, controlled by this extended conical intersection seam and that the shape of the conical intersection seam can be understood in terms of simple VB arguments. Further, the shape and energetics of the asynchronous segment of the conical intersection seam suggest that the 1,2 (ortho) and 1,3 (meta) products are equally preferred. The 1,4 (para) point on the conical intersection is higher in energy and corresponds to a local maximum on the seam.

In this paper, we describe the results of semiclassical dynamics simulations of photoinduced $[2+2]$ cycloaddition of benzene and ethylene. Specifically, we investigated the effect of the stacking orientation at the moment of laser excitation of the benzene molecule on the subsequent 


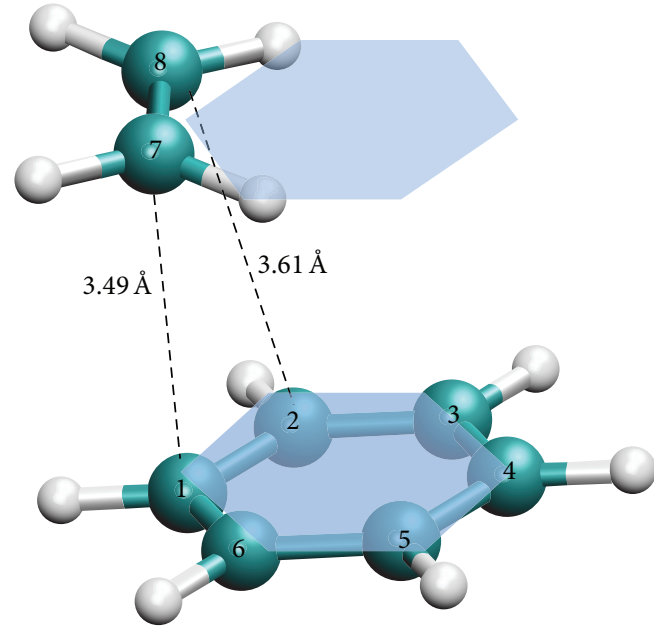

$\delta(8-7-1-2)=38.2^{\circ}$

(a)

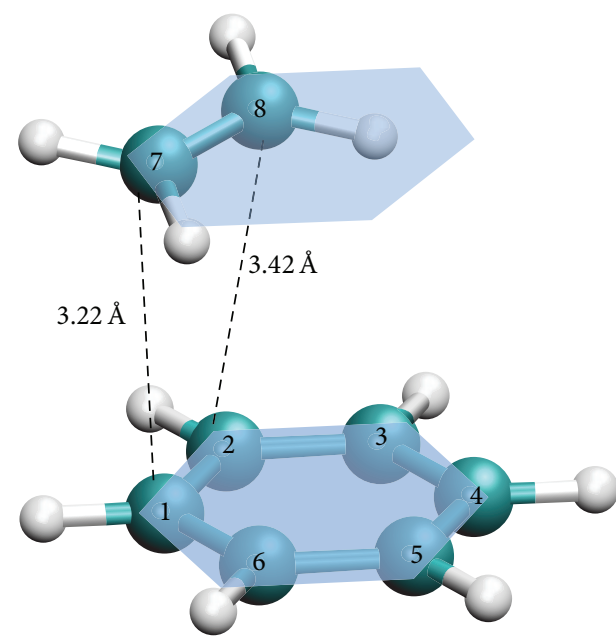

$\delta(8-7-1-2)=-23.6^{\circ}$

(b)

FIgure 1: (a) The equilibrated Geometry I and (b) equilibrated Geometry II of the benzene plus ethylene molecule with atomic labeling. Shadows herein indicate hypothetical hexagonal prism space of upward projection of benzene molecule.

dynamics. Previous studies on stacked DNA base molecules have shown that the initial orientation of reactant molecules affects the excited state dynamics [7-10]. Our simulations provide detailed dynamics features from photon excitation to the formation of product. The results are interpreted in terms of the geometry of conical intersection (CI) between the first singlet $\left(\mathrm{S}_{1}\right)$ excited state and ground state $\left(\mathrm{S}_{0}\right)$, which was optimized by using CASSCF method.

\section{Methodology}

2.1. Semiclassical Dynamics Simulation. We carried out the dynamics simulations using the semiclassical electron-radiation-ion dynamics (SERID) method. A detailed description of this technique has been published elsewhere $[11,12]$ and only a brief review is given here. In this approach, the valence electrons are calculated by the time-dependent Schrödinger equation while both the radiation field and the motion of the nuclei are treated by the classical approximation. The one-electron states are updated by solving the timedependent Schrödinger equation at each time step (typically 0.05 femtoseconds in duration) on a nonorthogonal basis using a unitary algorithm obtained from the equation for the time evolution operator [13]. The laser pulse is characterized by the vector potential $\mathbf{A}$, which is coupled to the Hamiltonian via the time-dependent Peierls substitution [14]. The Hamiltonian matrix elements, overlap matrix elements, and repulsive energy are calculated with the density-functional based tight bonding (DFTB) method $[15,16]$. The DFTB method does have essentially the same strengths and limitations as time-dependent density-functional theory (TDDFT). In particular, the bonding is well-described, but the excited-state energies are typically too low. For this reason, we matched the effective central photon energy of the laser pulse to the relevant density-functional (rather than experimental) excitation energy and this should not affect the interpretation of the results. In this technique, forces acting on nucleus or ions are computed by the Ehrenfest equation, which is obtained by neglecting the terms of second and higher order in the quantum fluctuations in the exact Ehrenfest theorem. The nuclear motion is numerically integrated with the velocity Verlet algorithm (which preserves phase space). A time step of 50 attoseconds was used based on the test of energy conservation.

The SERID method has been used in biologically relevant studies such as photoinduced dimerization of thymine [9] and cytosine [10] via $[2+2]$ photocycloaddition reaction. All the results were found to be consistent with experimental observations. The formation of cyclobutane pyrimidine dimer may provide good references for $[2+2]$ photocycloaddition of benzene and ethylene.

2.2. CASSCF. Multiconfigurational self-consistent field (MCSCF) is a method in quantum chemistry used to generate qualitatively correct reference states of molecules in cases where Hartree-Fock (HF) and DFT are not adequate (e.g., for molecular ground states which are quasi-degenerate with low-lying excited states or in bond breaking situations). It uses a linear combination of configuration state functions (CSF) or configuration determinants to approximate the exact electronic wavefunction of an atom or molecule. In an MCSCF calculation, the set of coefficients of both the CSFs or determinants and the basis functions in the molecular orbitals are varied to obtain the total electronic wavefunction with the lowest possible energy. This method can be considered a combination of configuration interaction (where the molecular orbitals are not varied but the expansion of the wave function) and Hartree-Fock (where 

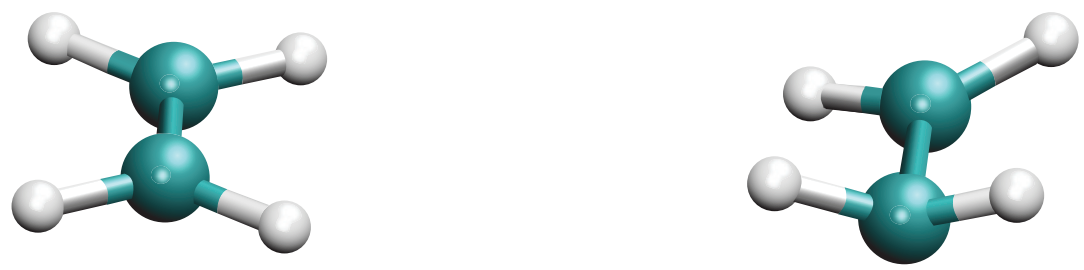

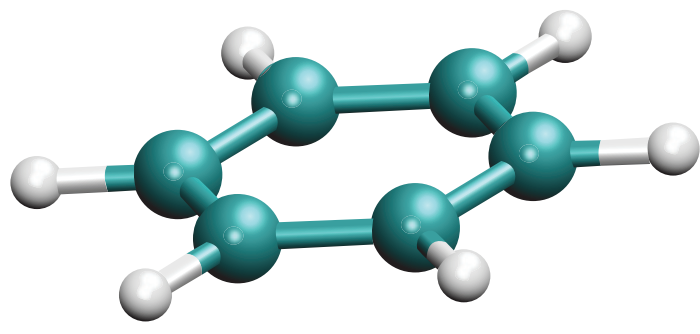

(a) $t=0 \mathrm{fs}$

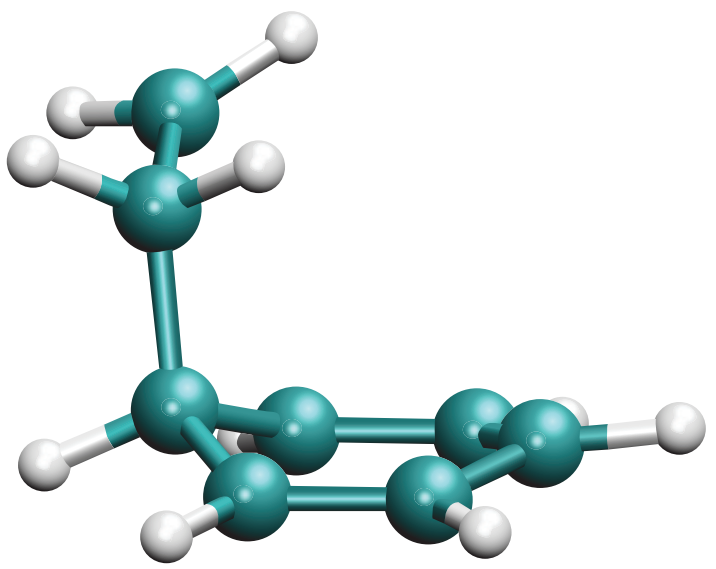

(c) $t=711 \mathrm{fs}$

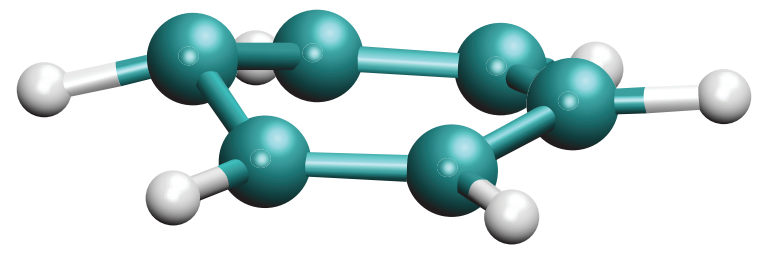

(b) $t=700 \mathrm{fs}$

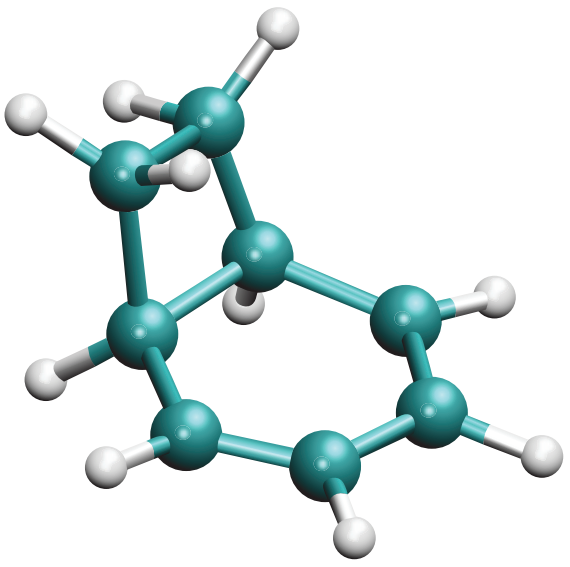

(d) $t=721 \mathrm{fs}$

FIGURE 2: Snapshots taken from the simulation of synchronous cycloaddition mode of stacked benzene and ethylene molecules at (a) 0 , (b) 700 , (c) 711 , and (d) $721 \mathrm{fs}$. The benzene molecule is subjected to the irradiation of a $25 \mathrm{fs}$ ( $\mathrm{fwhm}$ ) laser pulse with a fluence of $96.93 \mathrm{~J} \cdot \mathrm{m}^{-2}$ and photon energy of $4.7 \mathrm{eV}$.

there is only one determinant but the molecular orbitals are varied). A particularly important MCSCF approach is the CASSCF method, where the linear combination of CSFs includes all that arises from a particular number of electrons in a particular number of orbitals $[17,18]$.

In this research, the CASSCF computations used an 8electron and 8-orbital active space involving all the $\pi$ orbitals of benzene and ethylene moieties. All the geometries were optimized using the 6-31G(d) basis set in Gaussian 09 [19].

\section{Results and Discussion}

To obtain a ground state initial configuration, the simulation was run at room temperature for $1000 \mathrm{fs}$ starting with a randomly stacked geometry of benzene and ethylene. During this equilibration period, one hundred geometries were taken at equal time intervals; each geometry was subjected to a laser pulse. Figure 1 shows two representative geometries, at the moment of excitation, that lead to two different mechanisms.
In Figure 1(a), the ethylene molecule was placed in such a way that $\mathrm{C}_{1}-\mathrm{C}_{7}=3.49 \AA, \mathrm{C}_{2}-\mathrm{C}_{8}=3.61 \AA$, and dihedral angle $\mathrm{C}_{8}-\mathrm{C}_{7}-\mathrm{C}_{1}-\mathrm{C}_{2}=38.2^{\circ}$ (Geometry $\mathrm{I}$ ); the carbon atoms in the ethylene molecule are numbered $\mathrm{C}_{7}$ and $\mathrm{C}_{8}$. The $\mathrm{C}_{8}$ atom sticks out of the hexagonal prism defined by a vertical projection of benzene molecule (as indicated by the two shadows) through $\mathrm{C}_{7}$. In Figure $1(\mathrm{~b})$, benzene and ethylene molecule are oriented as follows: $\mathrm{C}_{1}-\mathrm{C}_{7}=3.22 \AA, \mathrm{C}_{2}-\mathrm{C}_{8}=$ $3.42 \AA$, and dihedral angle $\mathrm{C}_{8}-\mathrm{C}_{7}-\mathrm{C}_{1}-\mathrm{C}_{2}=-23.6^{\circ}$ (Geometry II). The negative dihedral angle suggests that the $\mathrm{C}_{8}$ atom of ethylene molecule is in the hypothetical hexagonal prism space.

A $25 \mathrm{fs}$ FWHM laser pulse with a Gaussian profile, with photon energy of $4.7 \mathrm{eV}$, was applied to this complex. A fluence between 0.07 and $0.15 \mathrm{~kJ} \cdot \mathrm{m}^{-2}$ was chosen for this study. Within this range, effective electronic excitation occurs, but the forces produced do not break any bonds. The 100 trajectories show that Geometry I would lead to synchronous addition and asynchronous mode frequently 

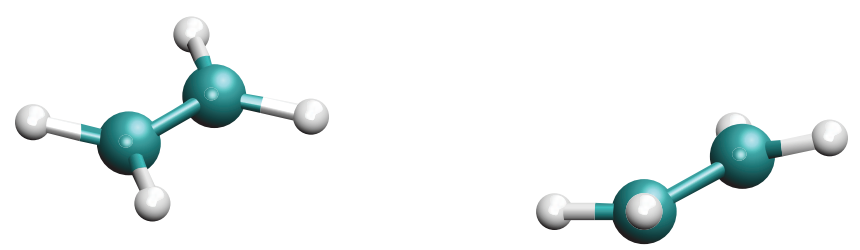

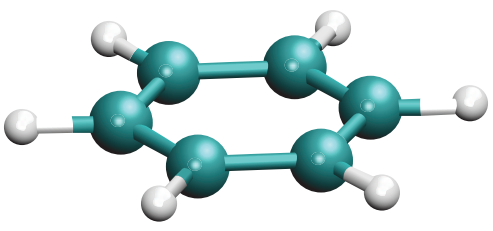

(a) $t=0 \mathrm{fs}$

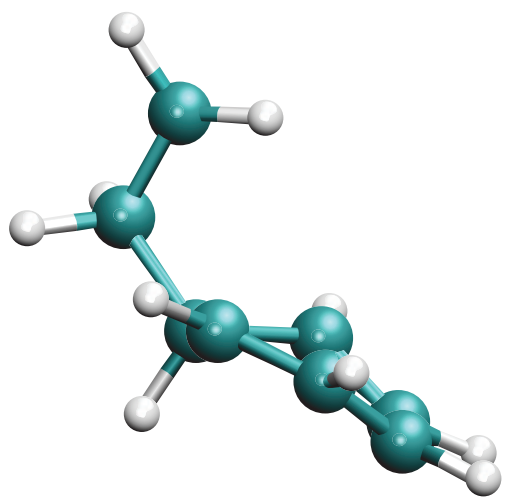

(d) $t=925 \mathrm{fs}$

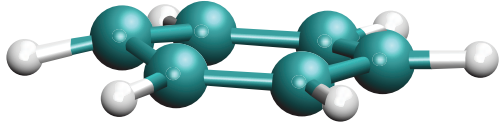

(b) $t=600 \mathrm{fs}$

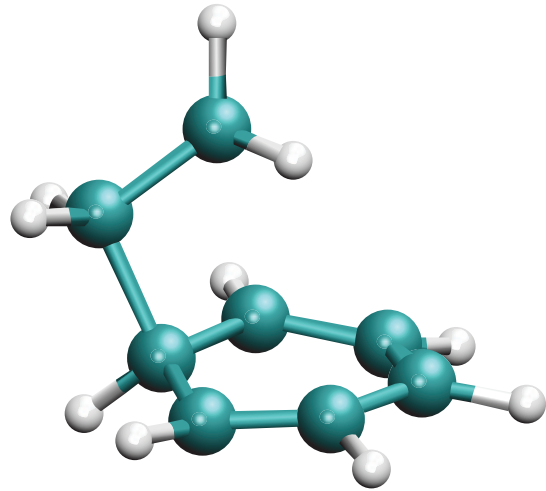

(e) $t=1615 \mathrm{fs}$

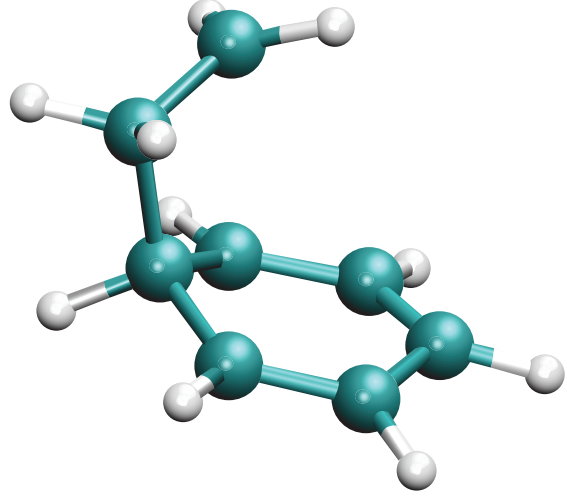

(c) $t=655 \mathrm{fs}$

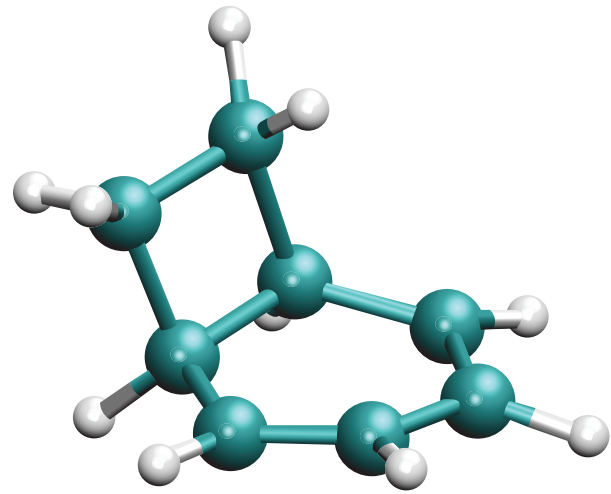

(f) $t=1715 \mathrm{fs}$

FIGURE 3: Snapshots taken from the simulation of asynchronous cycloaddition mode of stacked benzene and ethylene molecules at (a) 0, (b) 600 , (c) 655 , (d) 925 , (e) 1615 , and (f) 1715 fs. The benzene molecule is subjected to the irradiation of a $25 \mathrm{fs}$ (fwhm) laser pulse with a fluence of $94.11 \mathrm{~J} \cdot \mathrm{m}^{-2}$ and photon energy of $4.7 \mathrm{eV}$.

results from Geometry II. Here a typical trajectory for each case is reported in this paper.

Some snapshots of the synchronous and asynchronous $[2+2]$ photocycloaddition reaction are presented in Figures 2 and 3 , respectively. Figure 2(a) shows the equilibrium geometries of benzene and ethylene molecules in the electronic ground state at the moment of excitation. After application of the laser pulse, the benzene molecule is electronically excited and distorted, but the ethylene molecule retains its ground state geometry (Figure 2(b)). Excitation energy is transferred from benzene to ethylene; the two molecules approach each other due and are linked by the synchronous formation of the $\mathrm{C}_{1}-\mathrm{C}_{7}$ and $\mathrm{C}_{2}-\mathrm{C}_{8}$ bonds at $730 \mathrm{fs}$ (Figure 2(c)), and ultimately form the cyclobutane type product (Figure 2(d)). The structure of cyclobutane dimer remains stable through the end of the simulation. In Figure 3 , the $\mathrm{C}_{1}-\mathrm{C}_{7}$ bond is formed first (Figure 3(c)) to yield a so-called bonded intermediate (Figures $3(\mathrm{c})-3(\mathrm{e})$ ). The $\mathrm{C}_{2}-\mathrm{C}_{8}$ bond is later formed to yield the [2+2] product (Figure $3(\mathrm{f})$ ).

The HOMO and LUMO are $\pi$ and $\pi^{*}$ orbitals, respectively, and they are initially localized in benzene molecule as illustrated in Figure 4. The variations with time of the HOMO - 1, HOMO, LUMO and LUMO + 1 energies, and the time-dependent population of these molecular orbitals for the synchronous path are presented in Figure 5. Figure 5(a) shows that the LUMO energy decreases from -2.0 to $-2.8 \mathrm{eV}$ and the HOMO energy increases from -7.2 to $-7.0 \mathrm{eV}$ after laser excitation and remains more or less constant until $650 \mathrm{fs}$. At that point, the HOMO and LUMO energy gap narrows sharply to just $0.03 \mathrm{eV}$ at $715 \mathrm{fs}$; this near-degeneracy suggests an avoided crossing. Thereafter, the gap widens into an average of about $2.6 \mathrm{eV}$, suggesting the formation of the $[2+2]$ product. Figure $5(\mathrm{~b})$ shows that by the end of the laser pulse (which is $50 \mathrm{fs}$ ) about 1 electron is excited from the HOMO mainly to the LUMO. This excitation promotes the benzene molecule from the electronic ground state to a low-lying state $\pi \pi^{*}$. The coupling between the HOMO and LUMO at about 715 fs leads to a transfer of electrons from the LUMO to the HOMO. This deexcitation eventually brings the molecule from the low-lying $\pi \pi^{*}$ state to the electronic ground state. Shortly, after the avoided crossing, both the LUMO and HOMO levels move back toward their initial values.

Figure 5(c) shows that, in the asynchronous path, two nearly degenerate points are found at 655 and $920 \mathrm{fs}$, respectively. Figures 5(a) and 5(c) are nearly identical before $655 \mathrm{fs}$ and towards the end of the simulation (after $1600 \mathrm{fs}$ ). These suggest the same product formed, but different intermediates are involved in the asynchronous path. 

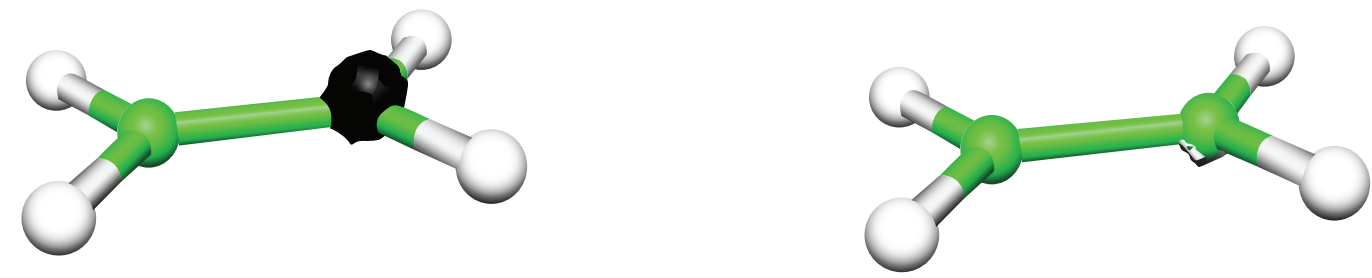

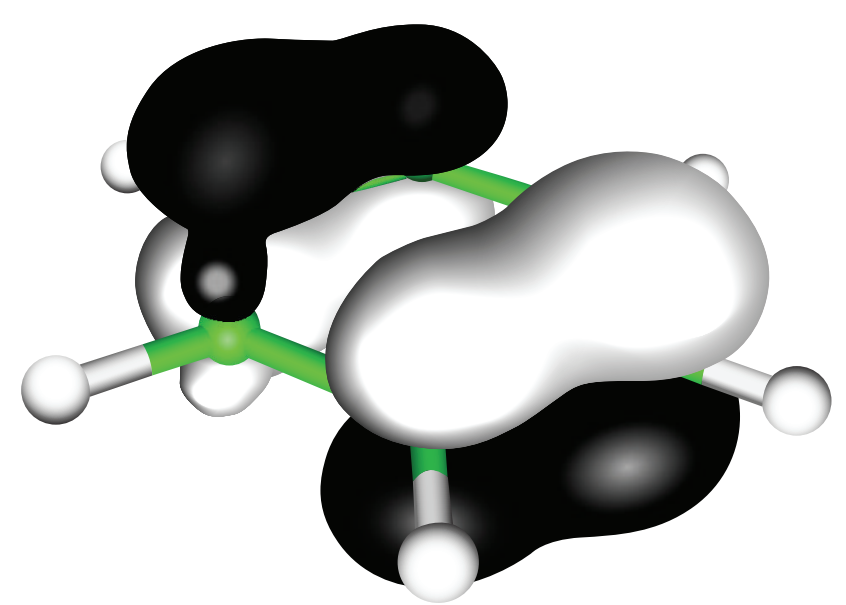

(a)

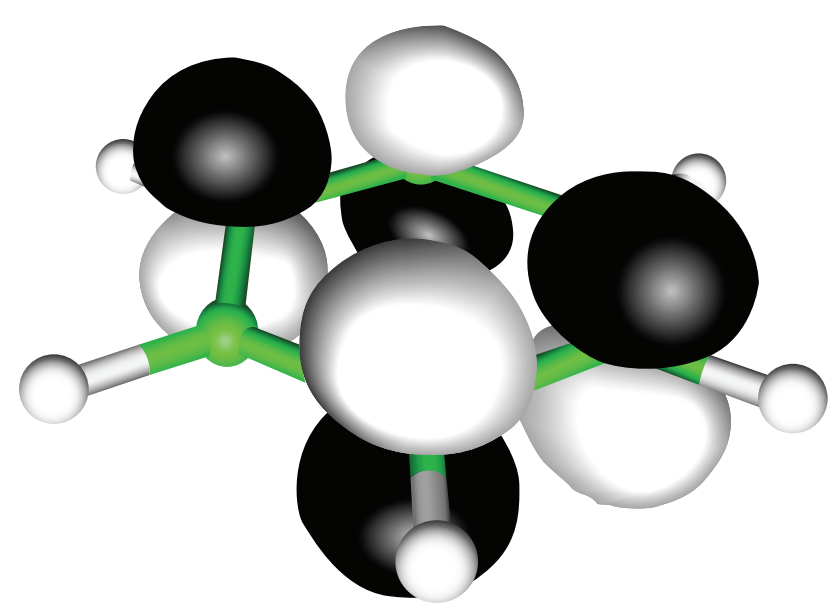

(b)

FIGURE 4: The diagrams for the HOMO and LUMO of benzene and ethylene molecules at the equilibrated structure computed by the DFTB approximation.

The variations with time of the lengths of the $\mathrm{C}_{1}-\mathrm{C}_{2}$ and $\mathrm{C}_{7}-\mathrm{C}_{8}$ bonds and $\mathrm{C}_{1}-\mathrm{C}_{7}$ and $\mathrm{C}_{2}-\mathrm{C}_{8}$ distances of both cases are presented in Figure 6 . The $\mathrm{C}_{1}-\mathrm{C}_{2}$ bond is part of a conjugated system and has a bond length of $1.40 \AA$ at the beginning of the simulation; its elongation to about $1.5 \AA$ after the laser pulse was applied suggests conversion to a single bond. Meanwhile, the $\mathrm{C}_{7}-\mathrm{C}_{8}$ bond length remains essentially constant, suggesting that the ethylene molecule remains in the ground state; only the benzene molecule is affected by the laser pulse. The approach of the excited benzene molecule causes changes in the ethylene as shown by increase in the $\mathrm{C}_{7}-\mathrm{C}_{8}$ bond length after $650 \mathrm{fs}$. Both of the $\mathrm{C}_{7}-\mathrm{C}_{8}$ and $\mathrm{C}_{1}-$ $\mathrm{C}_{2}$ bond lengths increase to $1.5 \AA$ after $720 \mathrm{fs}$, consistent with the formation of the cyclobutane dimer. The $\mathrm{C}_{1}-\mathrm{C}_{7}$ and $\mathrm{C}_{2}-$ $\mathrm{C}_{8}$ distances are initially 3.5 and $3.6 \AA$, respectively. They are more or less constant up to $100 \mathrm{fs}$, slowly decrease until $600 \mathrm{fs}$, and then sharply shorten to less than $1.6 \AA$ at about $700 \mathrm{fs}$ and $750 \mathrm{fs}$, respectively. These abrupt changes suggest that the formation of cyclobutane ring involves barrierless pathway. The formation of $\mathrm{C}_{1}-\mathrm{C}_{7}$ bond and $\mathrm{C}_{2}-\mathrm{C}_{8}$ bond is about $50 \mathrm{fs}$ apart in the simulation and would be difficult to distinguish experimentally. Thus, the formation of the two bonds may be regarded as a synchronous process.

Figure 7 shows the changes in the $\mathrm{C}_{3}-\mathrm{C}_{2}-\mathrm{C}_{6}-\mathrm{C}_{1}$ and $\mathrm{C}_{2}-\mathrm{C}_{1}-\mathrm{C}_{7}-\mathrm{C}_{8}$ dihedral angles with time. The former represents the out-of-plane vibration of benzene ring at $C_{1}$ position and the latter corresponds to an overlap of benzene and ethylene moieties. The $\mathrm{C}_{3}-\mathrm{C}_{2}-\mathrm{C}_{6}-\mathrm{C}_{1}$ dihedral angle changes indistinctively in synchronous addition mode, even at decay time. This indicates that deformation of $\mathrm{C}_{1}$ atom has a minimal role in the synchronous mechanism. On the other hand, in the asynchronous addition mode, one can observe a pyramidal geometry at the $\mathrm{C}_{1}$ site of benzene ring while the other five carbon atoms are almost planar, which resembles the configuration of $S_{1} / S_{0}$ conical intersection of benzene [20] at avoided crossing. It suggests that the out-of-plane vibration of the $\mathrm{C}_{1}$ atom plays a significant role in nonadiabatic coupling. In synchronous addition mode, the $\mathrm{C}_{2}-\mathrm{C}_{1}-\mathrm{C}_{7}-\mathrm{C}_{8}$ dihedral angle is always positive, which implies that $\mathrm{C}_{8}$ atom is outside the hexagonal prism space defined by the vertical projection of the benzene ring through $\mathrm{C}_{7}$, which leads to poor overlap between benzene and ethylene. However, in asynchronous addition mode, this angle has negative value throughout simulation. It suggests $\mathrm{C}_{8}$ atom is included in the virtual projection space.

The charge transfers for both cases are presented in Figure 8. During the simulation, no significant charge transfer is observed in the synchronous path. However, the benzene moiety loses about 0.7 electrons at $920 \mathrm{fs}$, and reversal occurs at $1600 \mathrm{fs}$ in asynchronous mode. This charge transfer at $920 \mathrm{fs}$ indicates that charge transfer plays an important role in the nonadiabatic coupling, which leads to a radiationless transition in asynchronous $[2+2]$ photocycloaddition. Furthermore, the charge transfer only occurred after electronic decay, suggesting a zwitterionic intermediate in ground state. 


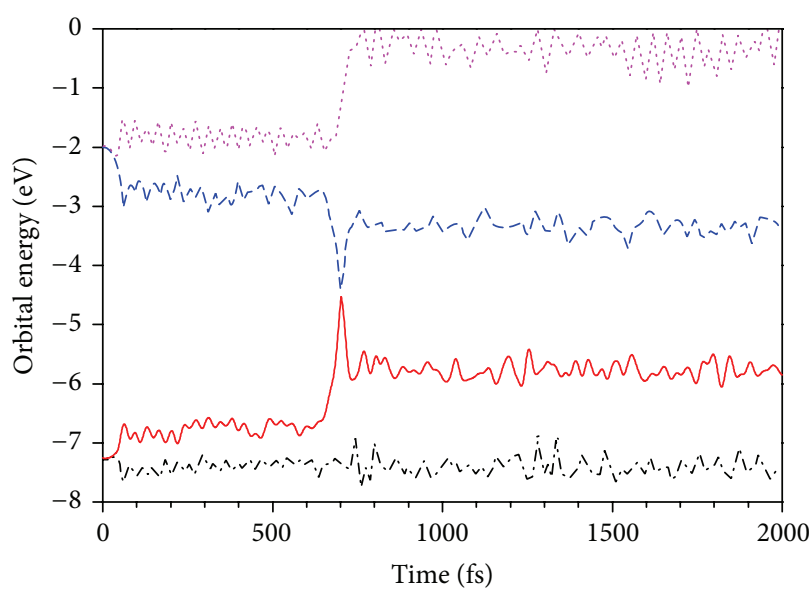

(a)

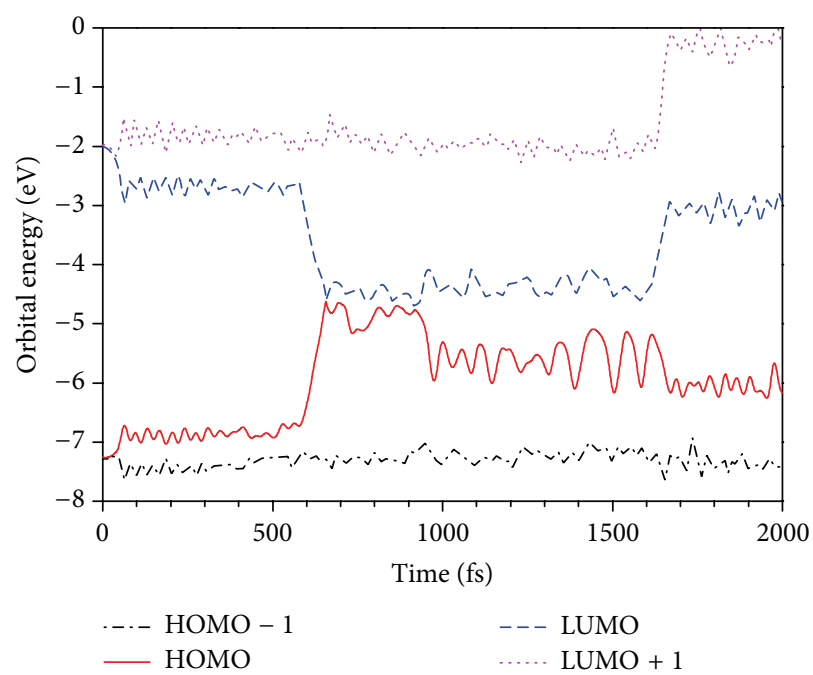

(c)

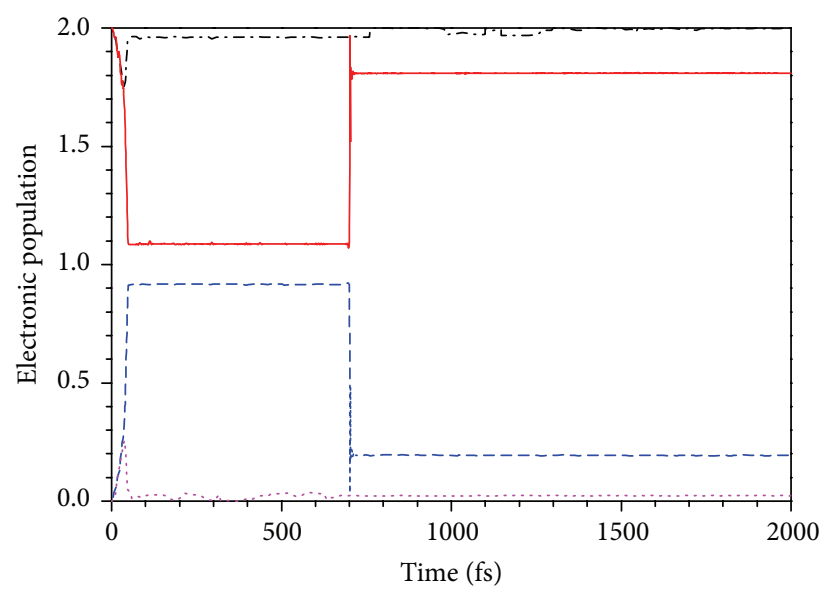

(b)

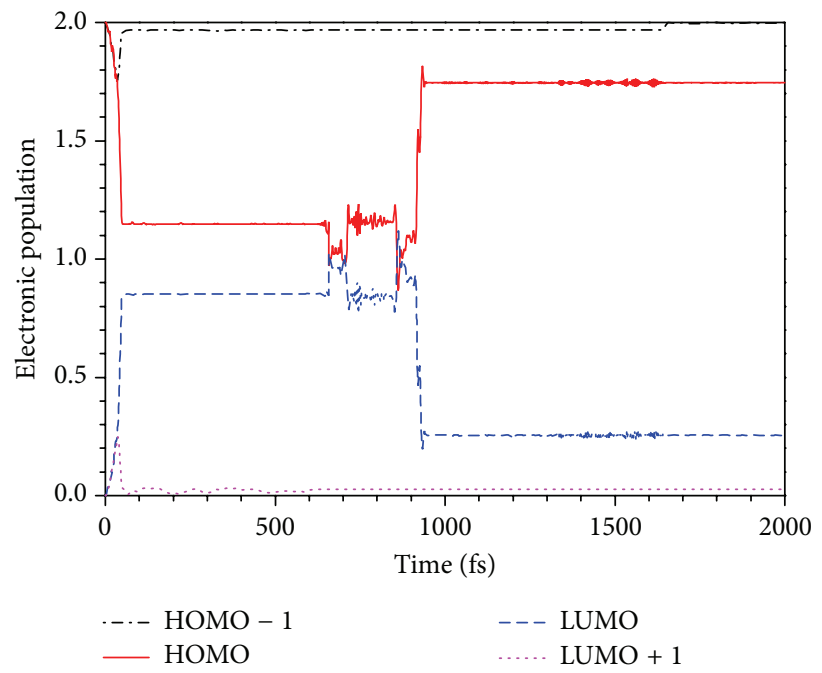

(d)

FIGURE 5: The variations with time of (a) the HOMO and LUMO energies; (b) the time-dependent population of stacked benzene and ethylene system in synchronous mode, which is subjected to a laser with frequency of $4.7 \mathrm{eV}$ and fluency of $96.93 \mathrm{~J} \cdot \mathrm{m}^{-2}$; (c) and (d) are the HOMO and LUMO energies and the time-dependent population in asynchronous mode, which is subjected to a laser with frequency of $4.7 \mathrm{eV}$ and fluence of $94.11 \mathrm{~J} \cdot \mathrm{m}^{-2}$.

Comparison of HOMO and LUMO energy gap with distance between $\mathrm{C}_{2}$ and $\mathrm{C}_{8}$ atoms in asynchronous mode is displayed in Figure 9. It is very clear that the energy gap goes through four stages. In stage 1 (50-650 fs), there is little interaction between two moieties because of the relatively large distance, as seen in Figure 6(d). In stage 2 (650-920 fs), $\mathrm{C}_{1}$ and $\mathrm{C}_{7}$ linking at $655 \mathrm{fs}$ leads to strong coupling of $\mathrm{HOMO}$ and LUMO and the gap sharply narrows to approximately zero. At this timescale, the minimum $\mathrm{C}_{2}-\mathrm{C}_{8}$ distance reaches $2.2 \AA$. In consideration of charge transfer not being observed, stage 2 may be defined as "biradical stage." In stage 3 (920$1600 \mathrm{fs}$ ), the energy gap increases to about $1 \mathrm{eV}$. It indicates that a new intermediate is formed. Indeed, stage 3 is in accord with charge transfer shown in Figure 8. The average of $\mathrm{C}_{2}-\mathrm{C}_{8}$ distance achieves $3.2 \AA$ and the minimum is $2.1 \AA$ during this range. Stage 3 is defined as zwitterionic. In stage 4 (>1600 fs), the electrons transfer back to benzene, and $\mathrm{C}_{2}-\mathrm{C}_{8}$ bond forms.
In consideration of shortcomings of SERID model, it is also valuable to investigate $S_{1} / S_{0}$ conical intersection in photocycloaddition reaction by using multiconfigurational self-consistent field method. The CASSCF ab initio computations used an 8-electron, 8-orbital active space involving all the $\pi$ orbitals of the benzene and ethylene moieties. It is difficult to optimize CI structure for a system of two interacting molecules because of the difficulty of making a good initial guess. To increase calculation efficiency, we used the geometry at decay time in simulation trajectory as the initial guess for $\mathrm{CI}$ optimization. All the $\mathrm{CI}$ geometries were optimized using the 6-31G(d) basis set. However, the equilibrated ground state structure of benzene plus ethylene from the simulation was used without any optimization. CASSCF results show that the two conical intersections corresponding to the synchronous and asynchronous mechanisms explicitly exist on the 6-31G(d) potential surface. Their energies (relative to benzene + ethylene in ground state) and some 

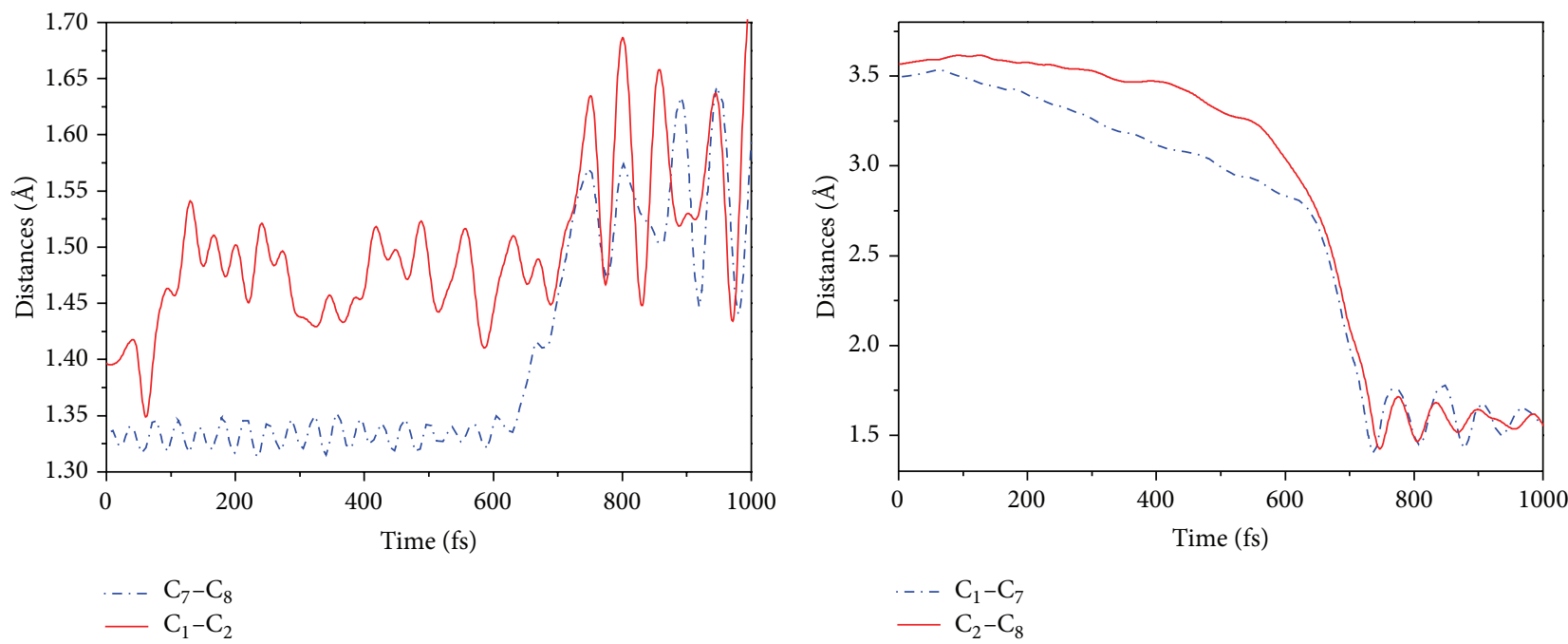

(a)

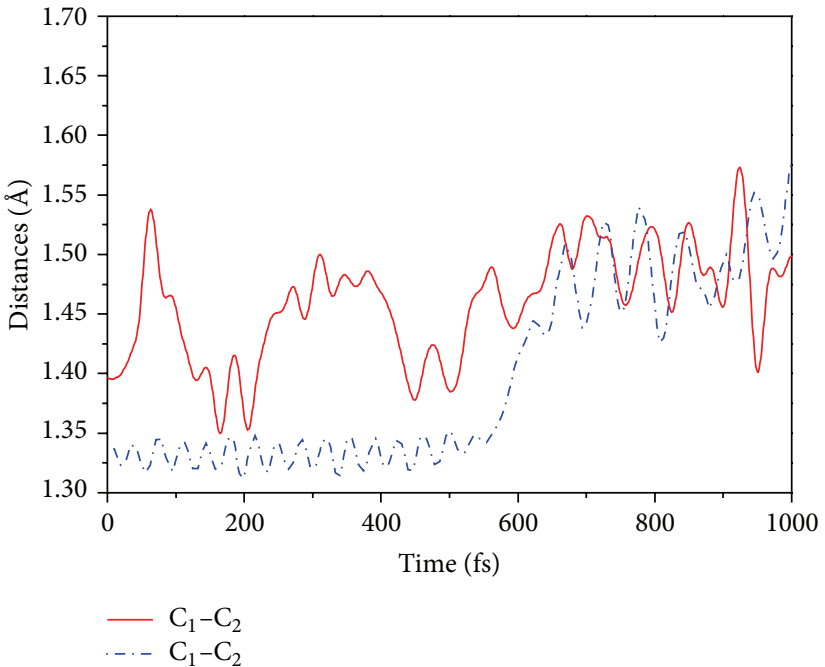

(c)

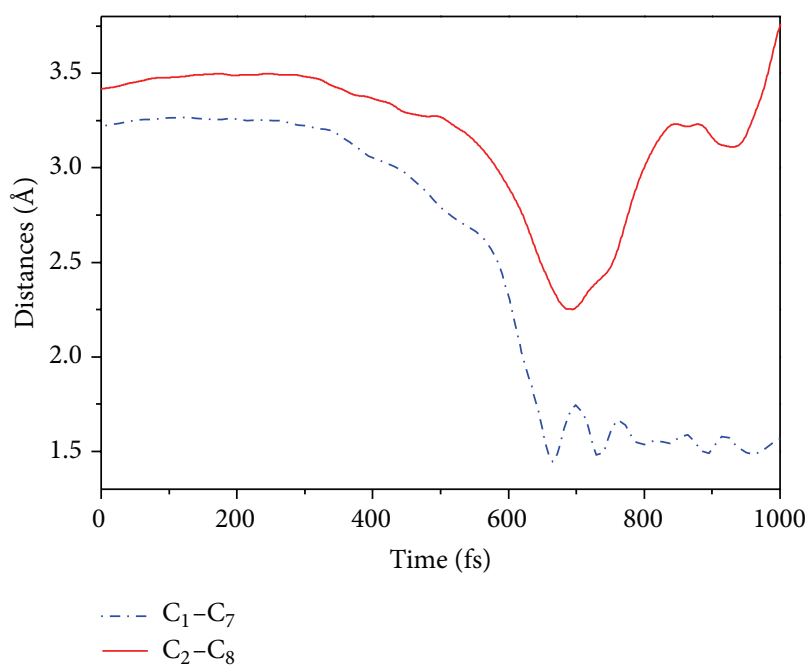

(d)

Figure 6: The variations of (a) $C_{1}-C_{2}$ and $C_{7}-C_{8}$ and (b) $C_{1}-C_{7}$ and $C_{2}-C_{8}$ distances with time of synchronous mode. (c) and (d) are these distances in asynchronous mode.

TABLE 1: Energies and geometries of both synchronous and synchronous [2 +2$]$ photocycloaddition reactions.

\begin{tabular}{|c|c|c|c|c|}
\hline Structure & Energy/eV & $\mathrm{C}_{1}-\mathrm{C}_{7} / \AA$ & $\mathrm{C}_{2}-\mathrm{C}_{8} / \AA$ & $\mathrm{C}_{2}-\mathrm{C}_{1}-\mathrm{C}_{7}-\mathrm{C}_{8} /^{\circ}$ \\
\hline $\mathrm{S}_{0}$ (syn) & 0 & 3.49 & 3.61 & 38.2 \\
\hline $\mathrm{S}_{0}$ (asyn) & 0.07 & 3.22 & 3.42 & -23.6 \\
\hline FC (syn) & 4.98 & - & - & - \\
\hline FC (asyn) & 4.99 & - & - & - \\
\hline CI (syn) & 5.76 & 2.10 & 2.06 & 15.2 \\
\hline CI (asyn) & 4.77 & 1.56 & 2.66 & -38.8 \\
\hline
\end{tabular}

significant geometrical parameters are presented in Table 1. The geometries of CIs, which are very similar to references $[5,6]$, are displayed in Figure 10.

Although Robb's group had calculated this system by using CASSCF, the overlaps of ethylene moiety and benzene ring in synchronous and asynchronous mode were neglected. Obviously, the $\mathrm{CI}_{(\text {syn) }}$ with a positive $\mathrm{C}_{2}-\mathrm{C}_{1}-\mathrm{C}_{7}-$ $\mathrm{C}_{8}$ dihedral of $15.2^{\circ}$ indicates an ineffective overlap between two moieties, compared to the initial simulated geometry (Figure 1(a)). In contrast, the $\mathrm{CI}_{(\text {syn) }}$ has better stacking, with a negative $\mathrm{C}_{2}-\mathrm{C}_{1}-\mathrm{C}_{7}-\mathrm{C}_{8}$ dihedral angle with value of $-38.8^{\circ}$. 


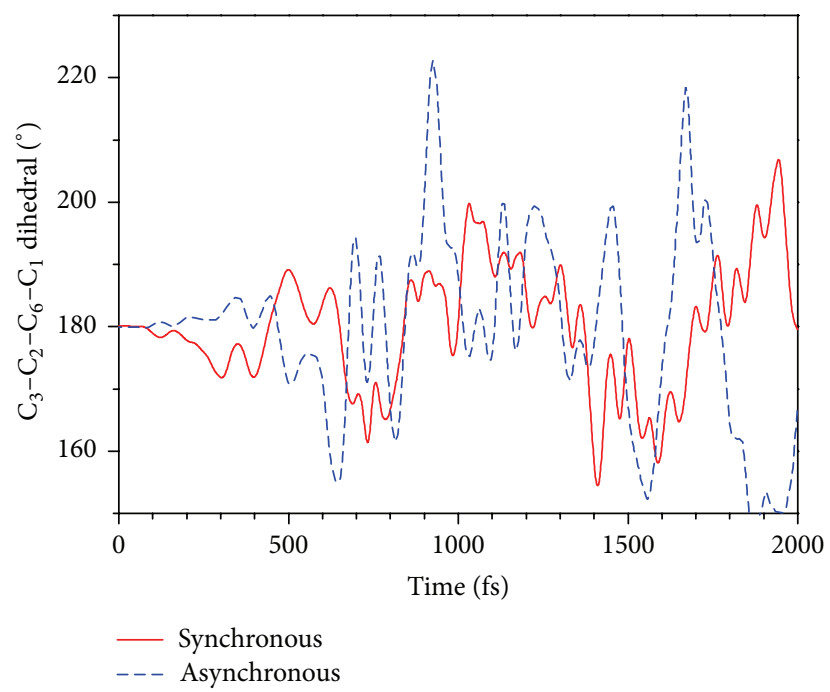

(a)

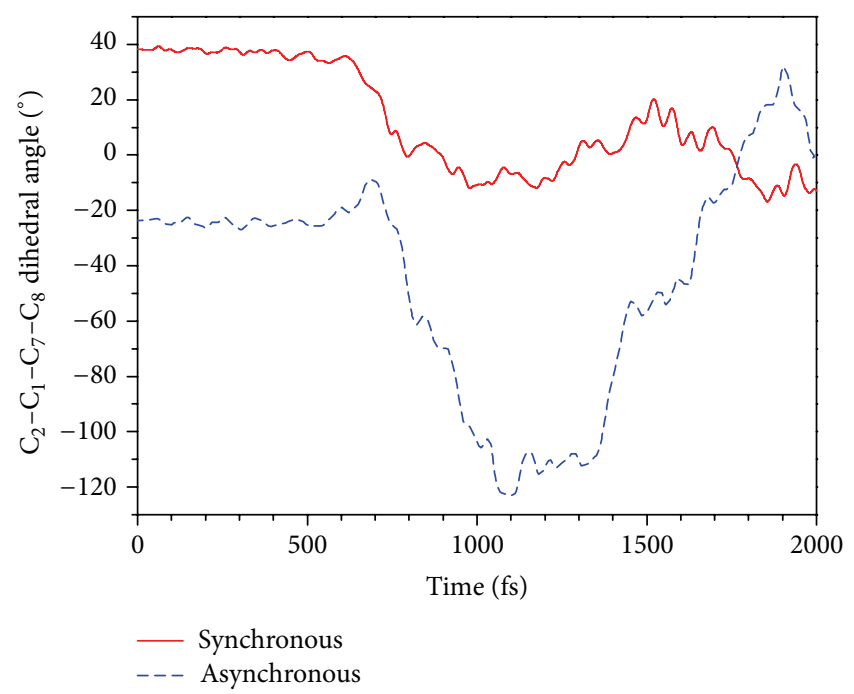

(b)

FIgURE 7: The variations of dihedral angles (a) $C_{3}-C_{2}-C_{6}-C_{1}$ and (b) $C_{2}-C_{1}-C_{7}-C_{8}$ with time for each mode.

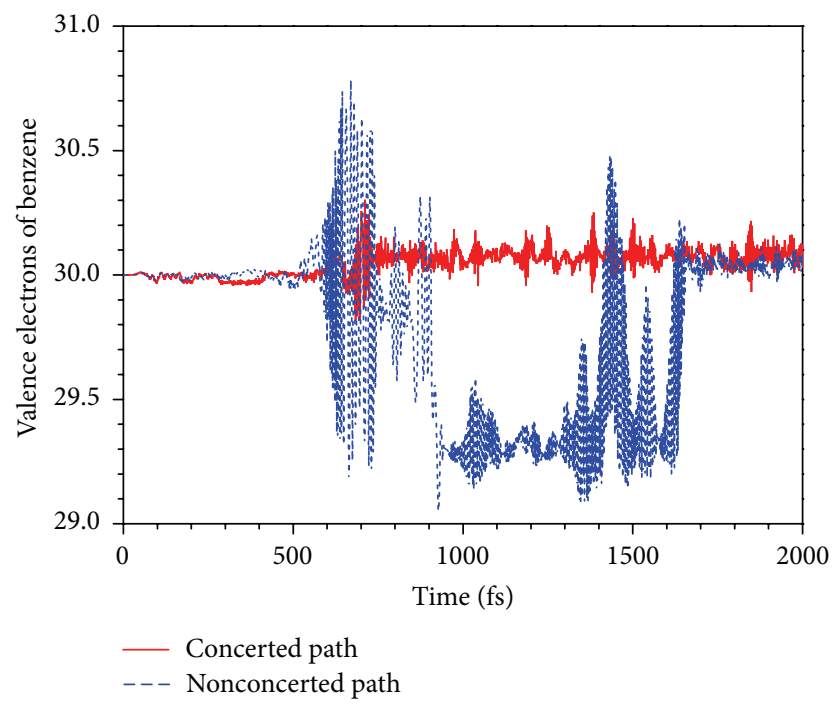

FIGURE 8: The variations of valence electrons of benzene molecule for each photocycloaddition mode.

The lower energy CI structure belongs to the asynchronous mode; the formation of the $\mathrm{C}_{1}-\mathrm{C}_{7}$ bond occurs without barrier to yield a low energy conical intersection. Given that benzene could form isomers such as benzvalene and fulvene via an $S_{1} / S_{0}$ conical intersection, the barrier for ethene + benzene cycloaddition must be very small to compete effectively with isomer formation. However, in synchronous mode, the CI energy is even higher than the vertical excitation energy at CASSCF $(8,8) / 6-31 G(d)$ level. This means that the synchronous photocycloaddition for benzene and ethylene system in $S_{1}$ excited state is less accessible.

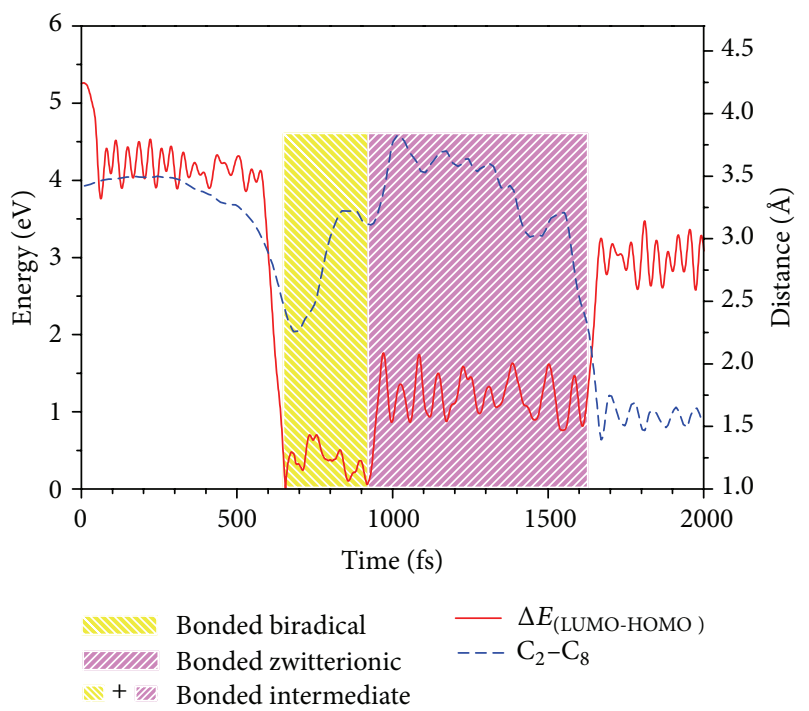

FIGURE 9: Comparison of HOMO and LUMO energy gap and $\mathrm{C}_{2}-\mathrm{C}_{8}$ distance for asynchronous mode.

\section{Conclusion}

In this paper, we report a semiclassical dynamics simulation study of $[2+2]$ cycloaddition reaction of benzene and ethylene induced by ultrashort laser pulses applied to the benzene molecule. When the ethylene molecule is within the vertical projection of benzene shape, excitation leads to a $\mathrm{C}_{1}-$ $\mathrm{C}_{7}$ bonded intermediate, which evolves to an electroneutral biradical and then to a zwitterionic intermediate at an avoided crossing. Eventually, the electron transfer is reversed as the cycloadduct is formed. Otherwise, a synchronous addition mechanism is observed. In the synchronous mode, the $\mathrm{C}_{1}-$ $\mathrm{C}_{7}$ and $\mathrm{C}_{2}-\mathrm{C}_{8}$ form chemical bond simultaneously and no charge transfer is observed. 


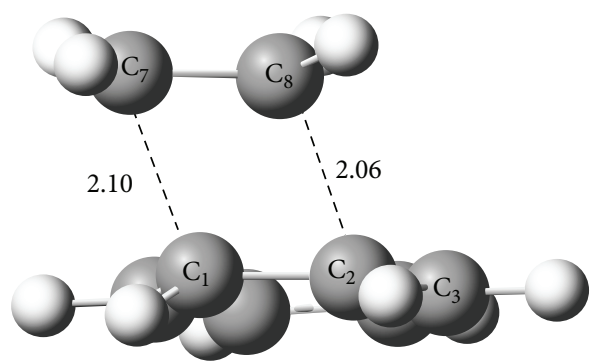

$\delta\left(\mathrm{C}_{8}-\mathrm{C}_{7}-\mathrm{C}_{1}-\mathrm{C}_{2}\right)=15.2^{\circ}$

(a)

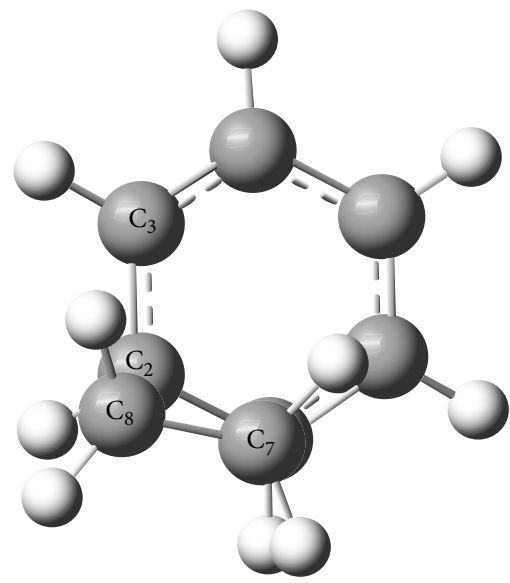

(c)

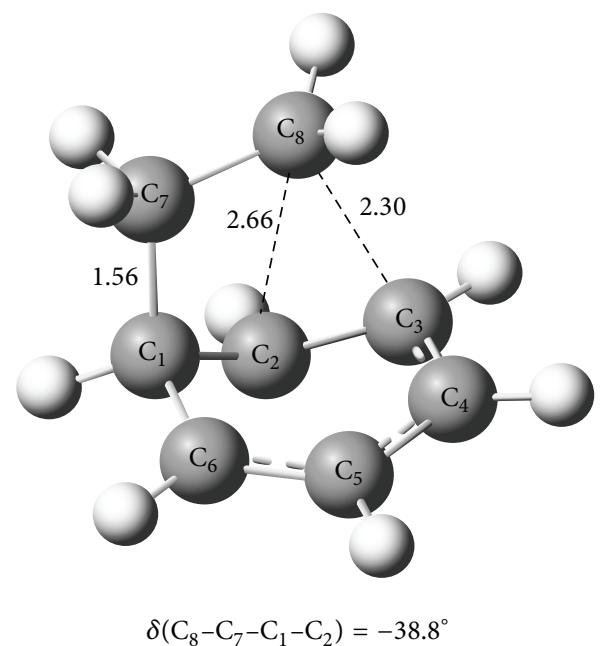

(b)

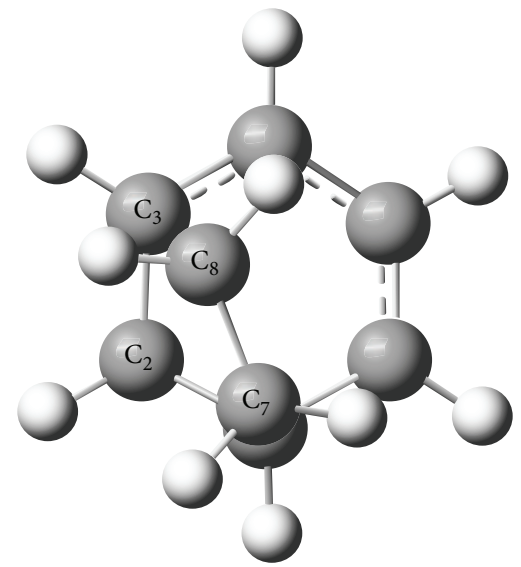

(d)

Figure 10: Optimized geometries of $S_{1} / S_{0}$ conical intersection of (a) synchronous and (c) asynchronous mode at CASSCF $(8,8) / 6-31 G(d)$ level. (b) and (d) are top view of (a) and (c), respectively.

Multiple trajectories simulations show that stacking effect plays an important role in [2+2] photocycloaddition reaction of benzene and ethylene molecule. Efficient stacked initial geometry will lead to asynchronous cycloaddition and inefficient stacked initial structure will result in synchronous photoreaction. Statistical results indicate that the quantum yield is higher for the asynchronous mechanism. The CASSCF calculations present that the evolution path from FC to CI is barrierless for asynchronous mode while it has to overcome $0.8 \mathrm{eV}$ barrier in synchronous addition reaction. It suggests asynchronous pathway is a favored predominant pathway for $[2+2]$ photocycloaddition reaction between benzene and ethylene, in accord with simulation results.

\section{Conflict of Interests}

The authors declare that there is no conflict of interests regarding the publication of this paper.

\section{Acknowledgments}

This work is supported by the Natural Science Foundation of Chongqing, China (no. CSTC2011jjA00009), and Project of the Science Technology Foundation of Chongqing Education Committee, China (nos. KJ120516 and KJ120521).

\section{References}

[1] U. Streit and C. G. Bochet, "The arene-alkene photocycloaddition," Beilstein Journal of Organic Chemistry, vol. 7, pp. 525-542, 2011.

[2] N. Hoffmann, "Photochemical reactions as key steps in organic synthesis," Chemical Reviews, vol. 108, no. 3, pp. 1052-1103, 2008.

[3] D. Bryce-Smith, "Orbital symmetry relationships for thermal and photochemical concerted cycloadditions to the benzene ring," Journal of the Chemical Society D, no. 14, pp. 806-808, 1969. 
[4] J. A. van der Hart, J. J. C. Mulder, and J. Cornelisse, "Funnels and barriers in the photocycloaddition of arenes to alkenes and dienes," Journal of Photochemistry and Photobiology A: Chemistry, vol. 86, no. 1-3, pp. 141-148, 1995.

[5] S. Clifford, M. J. Bearpark, F. Bernardi, M. Olivucci, M. A. Robb, and B. R. Smith, "Conical intersection pathways in the photocycloaddition of ethene and benzene: a CASSCF study with MMVB dynamics," Journal of the American Chemical Society, vol. 118, no. 31, pp. 7353-7360, 1996.

[6] J. J. Serrano-Pérez, F. de Vleeschouwer, F. de Proft, D. MendiveTapia, M. J. Bearpark, and M. A. Robb, "How the conical intersection seam controls chemical selectivity in the photocycloaddition of ethylene and benzene," The Journal of Organic Chemistry, vol. 78, no. 5, pp. 1874-1886, 2013.

[7] C. E. Crespo-Hernández, B. Cohen, and B. Kohler, "Base stacking controls excited-state dynamics in A.T DNA," Nature, vol. 436, no. 7054, pp. 1141-1144, 2005.

[8] C. T. Middleton, K. de la Harpe, C. Su, Y. K. Law, C. E. CrespoHernández, and B. Kohler, "DNA excited-state dynamics: from single bases to the double helix," Annual Review of Physical Chemistry, vol. 60, pp. 217-239, 2009.

[9] W. Zhang, S. Yuan, A. Li, Y. Dou, J. Zhao, and W. Fang, "Photoinduced thymine dimerization studied by semiclassical dynamics simulation," The Journal of Physical Chemistry C, vol. 114, no. 12, pp. 5594-5601, 2010.

[10] S. Yuan, W. Zhang, L. Liu, Y. Dou, W. Fang, and G. V. Lo, "Detailed mechanism for photoinduced cytosine dimerization: a semiclassical dynamics simulation," Journal of Physical Chemistry A, vol. 115, no. 46, pp. 13291-13297, 2011.

[11] Y. Dou, B. R. Torralva, and R. E. Allen, "Semiclassical electronradiation-ion dynamics (SERID) and cis-trans photoisomerization of butadiene," Journal of Modern Optics, vol. 50, no. 15-17, pp. 2615-2643, 2003.

[12] Y. Dou, B. R. Torralva, and R. E. Allen, "Interplay of electronic and nuclear degrees of freedom in a femtosecond-scale photochemical reaction," Chemical Physics Letters, vol. 392, no. 4-6, pp. 352-357, 2004.

[13] M. Lein, T. Kreibich, E. K. U. Gross, and V. Engel, "Strong-field ionization dynamics of a model $\mathrm{H}_{2}$ molecule," Physical Review A: Atomic, Molecular, and Optical Physics, vol. 65, Article ID 033403, 2002.

[14] S. Chelkowski, T. Zuo, O. Atabek, and A. D. Bandrauk, "Dissociation, ionization, and Coulomb explosion of $\mathrm{H}_{2}^{+}$in an intense laser field by numerical integration of the time-dependent Schrödinger equation," Physical Review A, vol. 52, no. 4, pp. 2977-2983, 1995.

[15] D. Porezag, T. Frauenheim, T. Köhler, G. Seifert, and R. Kaschner, "Construction of tight-binding-like potentials on the basis of density-functional theory: application to carbon," Physical Review B, vol. 51, no. 19, pp. 12947-12957, 1995.

[16] M. Elstner, D. Porezag, G. Jungnickel et al., "Self-consistentcharge density-functional tight-binding method for simulations of complex materials properties," Physical Review B, vol. 58, no. 11, pp. 7260-7268, 1998.

[17] J. Frank, Introduction to Computational Chemistry, John Wiley \& Sons, Chichester, UK, 2007.

[18] C. J. Cramer, Essentials of Computational Chemistry, John Wiley \& Sons, Chichester, UK, 2002.

[19] M. J. Frisch, G. W. Trucks, H. B. Schlegel et al., Gaussian 09, Revision D.01, Gaussian, Wallingford, Conn, USA, 2010.
[20] L. J. Palmer, L. N. Ragazos, F. Bernardi, M. Olivucci, and M. A. Robb, "An MC-SCF study of the S1 and S2 photochemical reactions of benzene," Journal of the American Chemical Society, vol. 115, no. 2, pp. 673-682, 1993. 

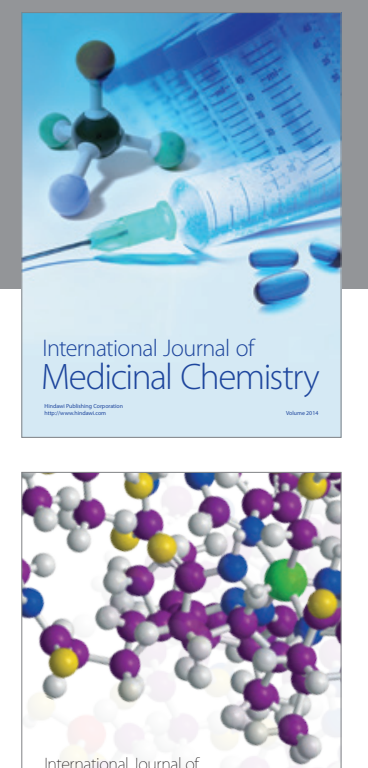

\section{Carbohydrate} Chemistry

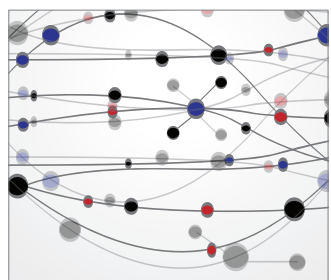

The Scientific World Journal
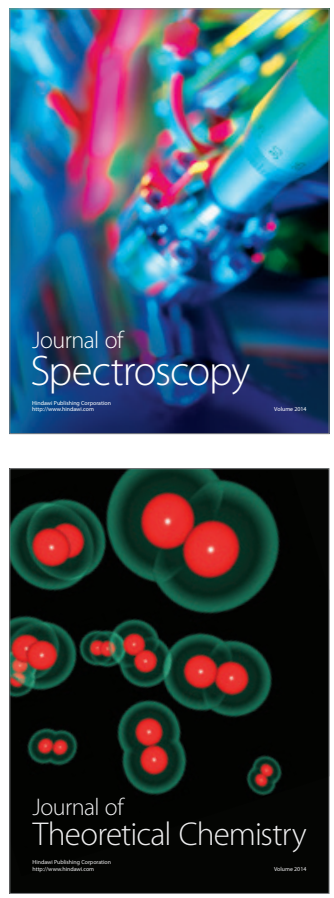
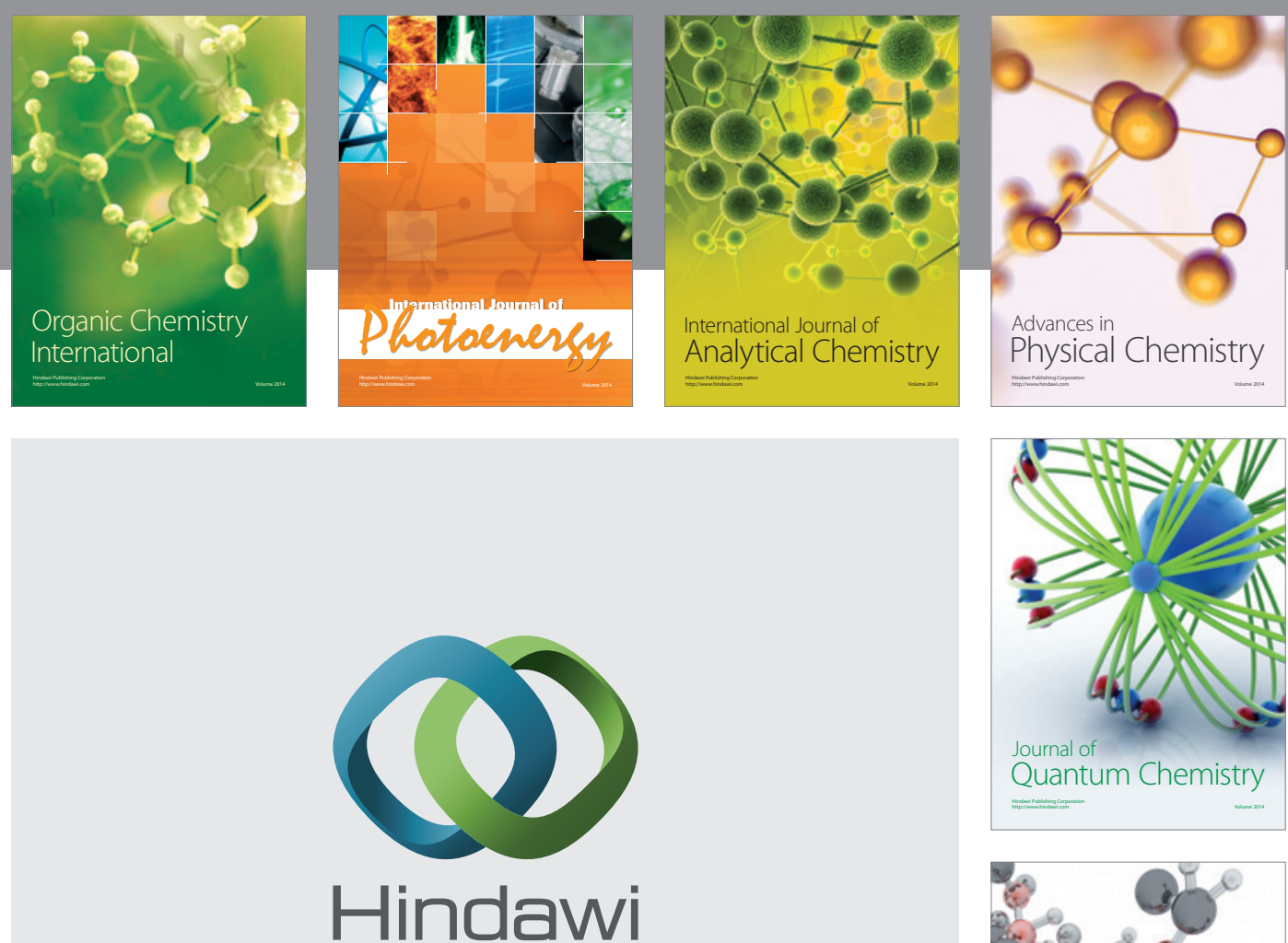

Submit your manuscripts at

http://www.hindawi.com

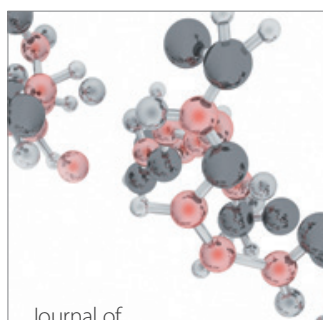

Analytical Methods

in Chemistry

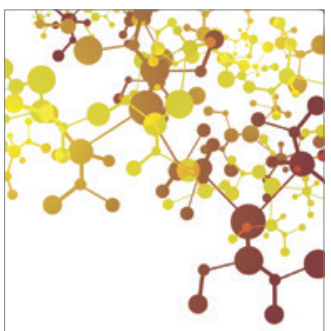

Journal of

Applied Chemistry

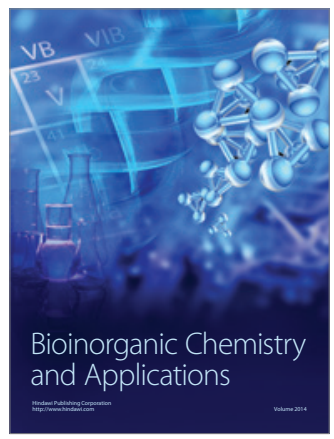

Inorganic Chemistry
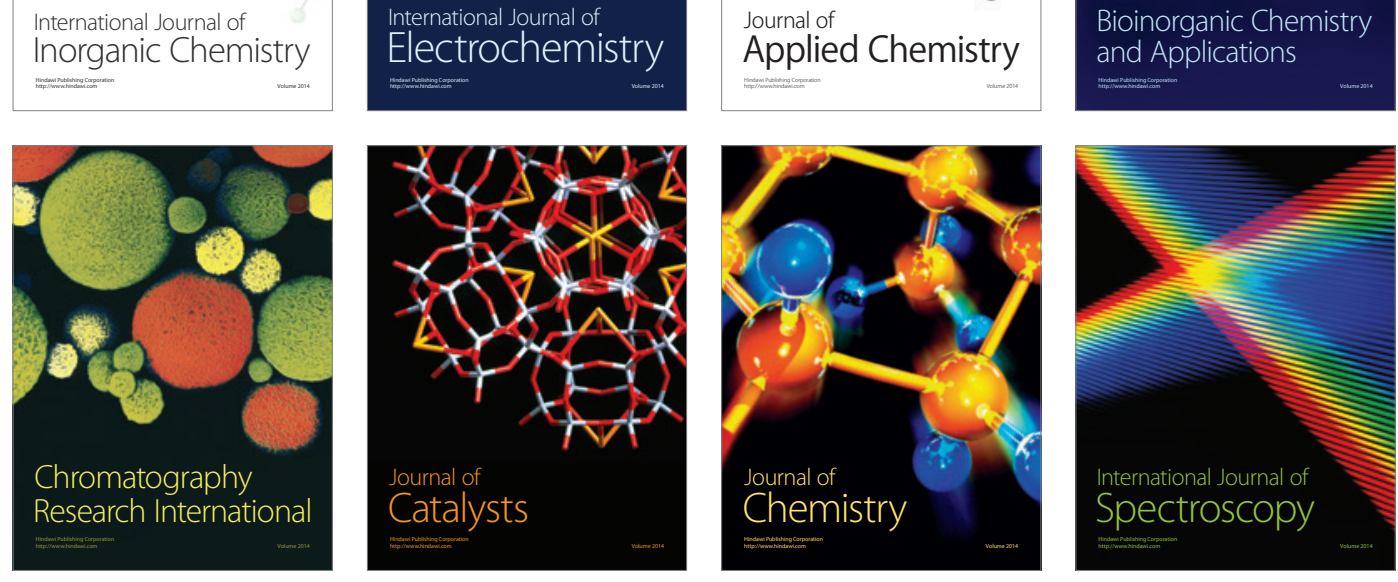